\title{
Testing the Latino paradox in Latin America: A population-based study of Intra-regional immigrants in Chile
}

\author{
BALTICA CABIESES ${ }^{1}$, HELENA TUNSTALL ${ }^{2}$, KATE PICKETT ${ }^{3}$
}

\section{ABSTRACT}

Background: Several studies in high-income countries report better health status of immigrants compared to the local population ("healthy migrant" effect), regardless of their socioeconomic deprivation. This is known as the Latino paradox. Aim: To test the Latino paradox within Latin America by assessing the health of international immigrants to Chile, most of them from Latin American countries, and comparing them to the Chilean-born. Material and Methods: Secondary data analysis of the population-based CASEN survey-2006. Three health outcomes were included: disability, illness/accident, and cancer/chronic condition (dichotomous). Demographics (age, sex, marital status, urban/rural, ethnicity), socioeconomic-status (SES: educational level, employment status and household income per-capita), and material standards (overcrowding, sanitation, housing quality). Crude and adjusted weighted regression models were performed. Results: One percent of Chile's population were immigrants, mainly from other Latin American countries. A "healthy migrant" effect appeared within the total immigrant population: this group had a significantly lower crude prevalence of almost all health indicators than the Chilean-born, which remained after adjusting for various demographic characteristics. However, this effect lost significance when adjusting by SES for most outcomes. The Latino paradox was not observed for international immigrants compared to the local population in Chile. Also, health of immigrants with the longest time of residency showed similar health rates to the Chilean-born. Conclusions: The Latino paradox was not observed in Chile. Protecting low SES immigrants in Chile could have large positive effects in their health at arrival and over time.

(Rev Med Chile 2013; 141: 1255-1265).

Key words: Emigrants and inmigrants; Health status; Human migration; Population; Socioeconomic factors.

\section{Evaluación de la "paradoja latina" en Chile utilizando datos de la encuesta de salud de 2006}

Antecedentes: Hay estudios que informan un mejor estado de salud de los inmigrantes en comparación con la población local (efecto del "migrante sano"), independientemente de su posición socioeconómica (PSE). Esto se conoce como la paradoja latina. Objetivo: Probar la paradoja latina dentro de América Latina en Chile. Material y Métodos: Análisis secundario de datos de la encuesta CASEN 2006. Tres resultados de salud se incluyeron: discapacidad, enfermedad/accidente, cáncer/ enfermedad crónica (variables dicotómicas). Se consideraron datos demográficos (edad, sexo, estado civil, zona urbana/rural, grupo étnico), PSE (nivel educativo,
${ }^{1} \mathrm{PhD}$, Lecturer at Universidad del Desarrollo, Chile; Visiting Scholar at University of York, England; ${ }^{2} \mathrm{PhD}$, Research Fellow at University of Edinburgh, Scotland;

${ }^{3} \mathrm{PhD}$, Professor of Epidemiology at University of York, England.

Conflicts of interest: None declared.

Received october 24, 2012; accepted august 2, 2013.

Correspondence to: Baltica Cabieses Address: Second floor, ARRC Building Department of Health Sciences University of York Heslington, York. Y010 5DD. UK E-mail: bcabieses@udd.cl; bbcv500@york.ac.uk 
situación laboral y el ingreso familiar per cápita), y condiciones materiales (hacinamiento, saneamiento, calidad de la vivienda). Modelos de regresión ponderados crudos y ajustados fueron analizados en STATA 11.0. Resultados: El uno por ciento de la población de Chile eran inmigrantes, principalmente de otros países de América Latina. Un efecto de "inmigrante sano" apareció dentro de la población inmigrante total: este grupo tenía una prevalencia cruda significativamente menor que la población chilena en todos los indicadores de salud. Sin embargo, este efecto de migrante sano pierde su significación al ajustar por PSE. Además, la salud de los inmigrantes con más tiempo de residencia mostró tasas similares de salud a la de origen chileno. Conclusiones: La paradoja latina no se observó en Chile. La protección de los inmigrantes de baja PSE podría tener grandes efectos positivos en su salud.

$M$ igration is a selective process and a social determinant of health ${ }^{1,2}$. People who move often are those who are more able to do so $^{3}$ and migrants represent a distinctive group from their home countries ${ }^{4}$. They often have better health status than both the population they leave behind and the population they arrive into in the receiving country $y^{5-7}$. This positive health selection of immigrants is known as the "healthy migrant" effect ${ }^{6,8}$. Evidence for this phenomenon suggests that first generation immigrants are often healthier than the people in their host country, as they report lower rates of several health outcomes than the local population ${ }^{9,10}$. Some of the strongest evidence for the healthy migrant effect is from research into the "Latino paradox"11,12. The Latino or Hispanic paradox refers to Hispanics and Latinos reporting health outcomes that are comparable to, or in some cases better than, those of their U.S. white counterparts, despite living in socioeconomic deprivation ${ }^{13-17,13-15,14,16,17}$. However, some authors have questioned the existence of healthy migrant effects and conflicting results have been found in some studies ${ }^{8,18}$.

Much less has been studied regarding the healthy migrant effect within Latin America ${ }^{8,19}$, despite the growing intra-regional mobility of people in this region ${ }^{20}$. This study describes the first large-scale quantitative analysis of the health of immigrants to Chile, most of them from other Latin American countries. Immigration to Chile has increased in recent years, reaching around $1.8 \%$ total population in 2007 ; the highest since the $1950 \mathrm{~s}^{21}$. Chile is an upper-middle-income country with a Gross Domestic Product per capita of USD $\$ 15866^{22}$. It has a population of just over 16 million and has experienced a progressive improvement of the health status of the population, a decline in the infant and general mortality rates, and an increase in life expectancy ${ }^{23,24}$.

There is conflicting evidence about the living condition and health status of international immigrants in Chile ${ }^{8,18}$. Some international research suggests that immigrants may have relatively good health despite poor living conditions (healthy migrant effect), while other evidence from qualitative research in Chile indicates that they may be at risk of poor health ${ }^{25,26}$. This study tested which of these contrasting findings best represents the experience of immigrants as a whole in Chile, by comparing the health status of immigrants with the Chileanborn population. We give special attention to socioeconomic conditions of immigrants in Chile and migration-related factors like country of origin and years living in the country. No study has yet tested the Latino paradox within the Latin American region. This analysis could shed some light on some of the characteristics, living conditions and health of Latino immigrants in Chile and in other similar countries in the region and elsewhere. It could also advance the discussion on the relevance of the comparison group when looking at migrant populations.

\section{Methods}

\section{Population and sample}

Secondary data analysis of the nationally representative CASEN (Caracterización SocioEconómica Nacional) survey $2006^{48}$.

The CASEN employed multistage probabilistic sampling with two phases (county and household), stratified by urban/rural. The sampling frame included all regions in Chile. The inclusion criteria for selection of counties were: (i) All urban 
counties with $>40,000$ inhabitants, (ii) All rural counties irrespective of the number of inhabitants, (iii) A random selection of a small proportion of counties $<40,000$ inhabitants. Twenty hard to reach counties were excluded, from a total of 605 counties.

The final sample consisted of 268,873 people in 73,720 households $(44,854$ urban and 28,866 rural ones, $95.4 \%$ of the total Chilean territory) ${ }^{27}$. The mean number of households included in the CASEN per region was representative of the total population within each region and also representative of the population in each urban and rural setting from each region ${ }^{28}$. The response rate was $84.8 \%{ }^{27}$.

\section{Migration status}

The survey asked: "in which country was your mother living when you were born?" Those who answered "in a different country from Chile" were identified as international immigrants, approximately $1 \%$ of the total sample $(\mathrm{n}=1877)$. An additional $0.7 \%$ preferred not to report migration status and since no other data suggested they were immigrants they were excluded from the analysis ( $\mathrm{n}=1477)$. It should be noted that the sum of immigrants plus the missing values were a fairly close representation of immigrants in Chile according to the latest statistics, around $1.8 \%{ }^{21}$.

\section{Self-reported health outcomes}

a. Disability in the past year: (yes/no) presence of one or more disabling conditions (visual/ hearing/speaking/physical/cognitive/psychiatric disability).

b. Health problem or accident in the past month (AHPA): (yes/no) presence of any health problem or accident in the past month.

c. Chronic condition or cancer (ACCC) in the past year: (yes/no), presence of this health event from any of the household members during the last 12 months.

\section{Demographic factors}

d. Age: continuous variable and categorical by three age-groups: $<15,15-64,>64$.

e. Sex (male/female).

f. Marital status: single, married/cohabitant, separated/divorced, widow.

g. Urban/rural area: as defined by the National Institute of Statistics ${ }^{28}$. h. Area of the country (northern/central/ southern).

i. Ethnicity: belonging to any of the nine legally recognised pre-Hispanic minority ethnic groups in Chile (yes/no).

Socioeconomic status (SES) Socioeconomic status is a complex and multidimensional concept ${ }^{29}$. The CASEN survey included the following:

a. Household income: total household income per capita in the past month in Chilean pesos and converted to USD purchasing power parity for 2006 (PPP, continuous variable, USD $\$ 1=$ 531 Chilean pesos, 2006 currency) ${ }^{22}$.

b. Educational level: the highest level achieved for the head of the household (university/ technical/high school/primary/none).

c. Employment status of the head of the household at the time of the interview (currently employed/unemployed).

\section{Material living standards}

Measured as recommended by the Chilean Ministry of Planning ${ }^{27}$ :

a. Housing quality: index that included the quality of the walls, floor and ceiling. Housing quality was categorised as poor (constructed of one or more non-enduring materials, such as plastic or cardboard, which is frequently used by those living in transient camps in Chile), regular (poor quality enduring materials) and high (high quality enduring materials).

b. Sanitary systems: defined as adequate when both a clean public water supply and a public sewage system were present or deficient if one or more of these measures were absent.

c. Household assets index (HAI): a continuous variable obtained from the combination of nine household assets through principal component analysis $(\mathrm{PCA})^{30}$ : car, washing machine, fridge, water heater, land phone, cable TV connection, computer, internet access, and mobile. Cronbach's alpha test and KaiserMeyer-Olkin measure of sampling adequacy were adequate $(0.81 \text { and } 0.88 \text {, respectively })^{31}$. The HAI accounted for $48 \%$ of total variance (range-1.00-9.87).

d. Overcrowding: binary variable as indicated by the Townsend score of deprivation ${ }^{32}$, which considers the ratio of the number of total rooms in the household over the total number 
of household members (a value of 1 indicates a ratio above 1 , that is more than 1 person per room in the building).

\section{Migration-related factors}

Country of origin (Perú/Argentina/Ecuador/ Bolivia/other; these categories were created given the high prevalence of Latin American immigrants in Chile, over $70 \%$ of the total immigrant population) and years living in the country (continuous variable, range $0-62$, and divided into six categories: less than a year, 1-5 years, 6-10 years, $11-15$ years, 16-20 years, and over 20 years). The "Other" category contained a mix of over 40 countries including other Latin American ones (Colombia/ Venezuela/Cuba/others), but also countries from other continents. As this category was a broad combination of multiple immigrants with very diverging backgrounds, results from them could be misleading and hence they were excluded from analysis.

\section{Analysis}

Descriptive statistics for each health outcome under study were reported as means (continuous variables) and proportions (categorical variables). Prevalence of each health outcome, crude and stratified by age groups, was also reported, with its corresponding Chi-square and t-tests for independent samples.

Odds Ratios (OR, logistic regressions) with their 95\% confidence intervals (robust standard errors method applied) were estimated by regression models. These models estimated the crude probability of presenting each health outcome and then adjusted by demographics, SES, material living standards, and migration-related factors in the immigrant and the Chilean-born populations. We also tested statistical significance of variables with more than two categories through the adjusted-Wald test.

Concerning post-estimation tests ${ }^{33,34}$, the Archer and Lemeshow goodness of fit test for a logistic regression model fitted using survey sample data was estimated (F-adjusted mean residual GOF test $)^{35}$. We studied confounding effects using its classic epidemiological definition ${ }^{36}$. Because of the large number of potential explanatory variables, testing of multiplicative interaction terms between significant co-variates for each regression model was primarily guided by theories of healthy migrant effect (i.e. push and pull theory ${ }^{37-39}$, cumulative causation theory $y^{40-43}$, globalization and migration $^{44-46}$ ). Rather than testing all possible interactions, these were restricted to terms of scientific interest and literature to support their exploration.

The CASEN 2006 survey was downloaded from a secured governmental Web page. Ethics approval was obtained from the Research Governance Committee at the University of York in july 2009. Data analyses were conducted with the STATA 11.0 statistical software package and estimations were weighted to take into account the survey's complex multistage sampling strategy and to attain population-based estimations ${ }^{47}$.

\section{Results}

\section{Crude prevalence of health outcomes}

There was a lower prevalence of any disability, AHPA, and ACCC in the immigrant population than the Chilean-born. There was the expected positive gradient in the prevalence of health problems by age among immigrants and the Chilean-born (Table 1). Immigrants showed great variation in their SES. There was an apparent wealthy group of immigrants, and a separate group of relatively poor immigrants, in low-status occupations, but not necessarily uneducated.

There was a significantly higher prevalence of any disability among immigrants with over 20 years living in Chile (11.60\% versus 3.55\%) and their rate was very similar to the Chilean-born population (Table 1).

\section{Partially adjusted models}

Partially adjusted models were estimated between the health problems and each set of covariates adjusting by age, sex and urban/rural (demographics/SES/material living standards/ migration-related factors).

Only a few covariates were associated with the health outcomes among immigrants in the partially adjusted models, possibly due to their great variability (Table 2). Overall, age and being employed were the most prominent factors associated with these health measures. Additionally, being a female $(\mathrm{OR}=2.78)$ and belonging to any ethnic group $(\mathrm{OR}=0.08)$ were significantly associated with ACCC. Living in rural areas was associated with the lower medical attentions $(\mathrm{OR}=0.67)$, and 
Testing the Latino paradox in Latin America - B. Cabieses et al

Table 1. Crude and stratified prevalence of health outcomes in the immigrant and the Chilean-born populations (CASEN survey 2006)

\begin{tabular}{|c|c|c|c|c|}
\hline \multirow[t]{2}{*}{$\begin{array}{l}\text { Crude and age-stratified rates } \\
\text { of six different health problems }\end{array}$} & \multicolumn{2}{|c|}{$\begin{array}{l}\text { CHILEAN-BORN POPULATION } \\
99 \% \text { total simple, } n=16130 \\
743 \text { weighted population } \\
\text { (266 } 966 \text { real observations) }\end{array}$} & \multicolumn{2}{|c|}{$\begin{array}{c}\text { IMMIGRANT POPULATION } \\
1 \% \text { total sample, } n=154431 \\
\text { weighted population } \\
\text { (1877 real observations) }\end{array}$} \\
\hline & $\%$ or mean & $95 \% \mathrm{Cl}$ & $\%$ or mean & $95 \% \mathrm{Cl}$ \\
\hline Any disability* & 6.93 & 6.74-7.13 & 3.55 & 2.49-5.02 \\
\hline $\begin{array}{l}\text { By age groups: } \\
\quad<16 \text { years old } \\
16-65 \text { years old* } \\
>65 \text { years old* }\end{array}$ & $\begin{array}{l}2.58 \\
6.21 \\
26.28\end{array}$ & $\begin{array}{c}2.36-2.82 \\
5.99-6.44 \\
25.34-27.24\end{array}$ & $\begin{array}{l}2.18 \\
2.96 \\
12.39\end{array}$ & $\begin{array}{l}0.74-6.23 \\
1.85-4.72 \\
7.04-20.90\end{array}$ \\
\hline $\begin{array}{l}\text { By country of origin: } \\
\text { Perú } \\
\text { Argentina } \\
\text { Ecuador } \\
\text { Bolivia } \\
\text { Other }\end{array}$ & $\begin{array}{l}- \\
- \\
- \\
- \\
-\end{array}$ & $\begin{array}{l}- \\
- \\
- \\
- \\
-\end{array}$ & $\begin{array}{l}1.45 \\
2.87 \\
3.58 \\
3.99 \\
1.89\end{array}$ & $\begin{array}{c}0.50-4.08 \\
1.78-4.59 \\
1.00-11.98 \\
1.37-11.05 \\
0.87-5.02\end{array}$ \\
\hline $\begin{array}{l}\text { By years living in Chile: } \\
<1 \text { year } \\
1-5 \text { years } \\
6-10 \\
11-15 \\
16-20 \\
>20 \text { years* }\end{array}$ & $\begin{array}{l}- \\
- \\
- \\
- \\
- \\
-\end{array}$ & $\begin{array}{l}- \\
- \\
- \\
- \\
- \\
-\end{array}$ & $\begin{array}{c}2.49 \\
1.37 \\
3.21 \\
0.81 \\
0.32 \\
11.60\end{array}$ & $\begin{array}{l}1.27-4.80 \\
0.32-5.73 \\
1.21-8.29 \\
0.19-3.48 \\
0.07-1.35 \\
7.14-18.29\end{array}$ \\
\hline Any health problem/ accident* & 15.72 & 15.41-16.03 & 10.80 & $8.70-13.32$ \\
\hline $\begin{array}{l}\text { By age groups: } \\
<16 \text { years old* } \\
16-65 \text { years old* } \\
>65 \text { years old }\end{array}$ & $\begin{array}{l}14.94 \\
14.29 \\
30.05\end{array}$ & $\begin{array}{l}14.38-15.51 \\
13.95-14.63 \\
29.05-31.07\end{array}$ & $\begin{array}{l}6.92 \\
10.44 \\
21.36\end{array}$ & $\begin{array}{l}3.80-12.28 \\
8.25-13.13 \\
11.00-37.38\end{array}$ \\
\hline $\begin{array}{l}\text { By country of origin: } \\
\text { Perú } \\
\text { Argentina } \\
\text { Ecuador } \\
\text { Bolivia }\end{array}$ & $\begin{array}{l}- \\
- \\
-\end{array}$ & $\begin{array}{l}- \\
- \\
-\end{array}$ & $\begin{array}{l}11.11 \\
13.21 \\
9.00 \\
8.43\end{array}$ & $\begin{array}{l}7.89-15.43 \\
9.38-18.28 \\
2.05-31.84 \\
3.59-18.53\end{array}$ \\
\hline $\begin{array}{l}\text { By years living in Chile: } \\
<1 \text { year } \\
1-5 \text { years } \\
6-10 \\
11-15 \\
16-20 \\
>20 \text { years }\end{array}$ & $\begin{array}{l}- \\
- \\
- \\
- \\
- \\
-\end{array}$ & $\begin{array}{l}- \\
- \\
- \\
- \\
- \\
-\end{array}$ & $\begin{array}{l}10.56 \\
7.97 \\
9.66 \\
4.70 \\
11.08 \\
18.43\end{array}$ & $\begin{array}{c}6.84-15.96 \\
4.44-13.19 \\
6.02-15.16 \\
1.67-12.57 \\
5.37-21.46 \\
12.51-26.32\end{array}$ \\
\hline Any chronic condition or cancer* & 5.85 & $5.68-6.02$ & 3.90 & $2.68-5.63$ \\
\hline $\begin{array}{l}\text { By age groups: } \\
<16 \text { years old* } \\
16-65 \text { years old* } \\
>65 \text { years old }\end{array}$ & $\begin{array}{l}1.92 \\
5.37 \\
21.88\end{array}$ & $\begin{array}{c}1.71-2.14 \\
5.18-5.56 \\
20.96-22.82\end{array}$ & $\begin{array}{l}0.003 \\
2.83 \\
22.61\end{array}$ & $\begin{array}{c}0.00-0.28 \\
1.84-4.33 \\
11.94-38.63\end{array}$ \\
\hline $\begin{array}{l}\text { By country of origin: } \\
\text { Perú } \\
\text { Argentina } \\
\text { Ecuador } \\
\text { Bolivia } \\
\text { Other }\end{array}$ & $\begin{array}{l}- \\
- \\
- \\
- \\
-\end{array}$ & $\begin{array}{l}- \\
- \\
- \\
- \\
-\end{array}$ & $\begin{array}{l}1.67 \\
3.21 \\
2.02 \\
2.76 \\
5.21\end{array}$ & $\begin{array}{c}0.82-3.40 \\
1.77-5.75 \\
0.54-7.18 \\
0.46-14.75 \\
1.21-9.54\end{array}$ \\
\hline $\begin{array}{l}\text { By years living in Chile: } \\
<1 \text { year } \\
1-5 \text { years } \\
6-10 \\
11-15 \\
16-20 \\
>20 \text { years }\end{array}$ & $\begin{array}{l}- \\
- \\
- \\
- \\
- \\
-\end{array}$ & $\begin{array}{l}- \\
- \\
- \\
- \\
- \\
-\end{array}$ & $\begin{array}{l}4.03 \\
1.98 \\
2.56 \\
5.19 \\
2.04 \\
7.69\end{array}$ & $\begin{array}{c}1.76-8.99 \\
0.59-6.47 \\
1.18-5.54 \\
1.86-13.54 \\
0.66-6.09 \\
4.38-13.15\end{array}$ \\
\hline
\end{tabular}

${ }^{*} p$ value $<0.001$ when comparing the same category between the Chilean-born and the immigrant populations ( $\chi^{2}$ test or t-test for independent samples). 
Testing the Latino paradox in Latin America - B. Cabieses et al

Table 2. Odds Ratio (OR) of presenting different health outcomes in the immigrant population, adjusted by different sets of covariates separately (CASEN survey 2006)

\begin{tabular}{|c|c|c|c|c|c|c|}
\hline & \multicolumn{2}{|c|}{ Any disability } & \multicolumn{2}{|c|}{$\begin{array}{l}\text { Any health problem } \\
\text { or accident }\end{array}$} & \multicolumn{2}{|c|}{$\begin{array}{l}\text { Any chronic condition } \\
\text { or cancer }\end{array}$} \\
\hline & OR & 95\% Cl & OR & $95 \% \mathrm{Cl}$ & OR & $95 \% \mathrm{Cl}$ \\
\hline \multicolumn{7}{|l|}{ DEMOGRAPHIC SDH } \\
\hline Age & $1.04^{*}$ & $1.02-1.06$ & 1.02 & $0.96-1.06$ & $1.05^{*}$ & $1.02-1.08$ \\
\hline Sex $($ female $=1)$ & 0.56 & $0.25-1.25$ & 2.10 & $0.84-5.22$ & $2.78^{* *}$ & $1.26-6.71$ \\
\hline \multicolumn{7}{|l|}{ Marital status: } \\
\hline Single & 1.00 & - & 1.00 & - & 1.00 & - \\
\hline Married & 0.79 & $0.29-2.17$ & 2.05 & $0.82-5.13$ & 3.76 & $0.25-54.76$ \\
\hline Divorced & 2.57 & $0.52-8.73$ & 3.84 & $0.86-17.00$ & 5.20 & $0.15-17.21$ \\
\hline Widow & 1.07 & $0.26-4.39$ & 0.61 & $0.04-8.52$ & - & - \\
\hline Ethnicity: any & 1.06 & $0.17-6.48$ & 0.60 & $0.06-5.59$ & $0.08 * *$ & $0.008-0.07$ \\
\hline Zone (Rural = 1) & 1.56 & $0.80-3.04$ & 1.96 & $0.42-9.08$ & 0.33 & $0.04-6.28$ \\
\hline \multicolumn{7}{|l|}{ Area: } \\
\hline Northern & 1.00 & - & 1.00 & - & 1.00 & - \\
\hline Central & 0.48 & $0.14-1.64$ & 1.35 & $0.44-4.18$ & 0.57 & $0.21-1.52$ \\
\hline Southern & 0.89 & $0.27-2.91$ & 0.44 & $0.06-2.99$ & 0.93 & $0.21-1.52$ \\
\hline GOF test & - & $<0.001$ & - & $<0.001$ & - & $<0.001$ \\
\hline \multirow{2}{*}{\multicolumn{7}{|c|}{ SES (adjusted by age, sex and urban/rural) }} \\
\hline Educational level: & & & & & & \\
\hline No education & 1.94 & $0.41-9.12$ & 0.10 & $0.002-4.73$ & 0.03 & $0.001-0.89$ \\
\hline Primary School & 1.95 & $0.70-5.40$ & 0.78 & $0.21-2.80$ & 0.10 & $0.05-1.90$ \\
\hline High Schoolb & 1.05 & $0.37-2.91$ & 1.006 & $0.33-2.98$ & 0.78 & $0.23-2.62$ \\
\hline Technical level & 0.07 & $0.01-0.48$ & 0.50 & $0.10-2.45$ & 0.48 & $0.08-2.85$ \\
\hline University level & 1.00 & - & 1.00 & - & 1.00 & - \\
\hline Household income per capita (USD) & 0.68 & $0.18-2.51$ & 0.95 & $0.13-6.76$ & 0.84 & $0.12-1.52$ \\
\hline Current worker & 4.31 & $0.43-9.63$ & $0.13^{* *}$ & $0.03-0.52$ & $0.56^{*}$ & $0.09-0.98$ \\
\hline GOF test & - & $<0.001$ & - & $<0.001$ & - & $<0.001$ \\
\hline MATERIAL HOUSEHOLD CONDI & NS (adj & ted by age, & ex and ut & in/rural) & & \\
\hline Quality Household: & & & & & & \\
\hline Acceptable & 1.00 & - & 1.00 & - & 1.00 & - \\
\hline Sub-standard & 0.90 & $0.44-1.81$ & 1.98 & $0.89-4.40$ & 0.78 & $0.15-2.01$ \\
\hline Unfit & 4.37 & $0.86-22.01$ & 6.07 & $0.50-73.08$ & 0.63 & $0.06-6.01$ \\
\hline Sanitary Index (adequate $=1$ ) & 0.82 & $0.37-1.81$ & 4.18 & $0.89-19.63$ & 3.17 & $0.07-12.66$ \\
\hline Overcrowding & 0.58 & $0.26-1.30$ & 0.97 & $0.37-2.50$ & 0.55 & $0.02-12.54$ \\
\hline HAl & 0.94 & $0.87-1.07$ & 1.09 & $0.95-1.26$ & 0.76 & $0.51-1.12$ \\
\hline GOF test & - & $<0.001$ & - & $<0.001$ & - & $<0.001$ \\
\hline \multirow{2}{*}{\multicolumn{7}{|c|}{ MIGRATION-RELATED SDH (adjusted by age, sex and urban/rural) }} \\
\hline Years living in Chile & & & & & & \\
\hline Less than a year & 1.00 & - & 1.00 & - & 1.00 & - \\
\hline 1 to 5 years & 0.76 & $0.14-4.09$ & 0.88 & $0.37-1.75$ & 0.97 & $0.24-3.25$ \\
\hline 6 to 10 years & 1.72 & $0.48-6.17$ & 1.005 & $0.53-1.89$ & 1.38 & $0.35-5.41$ \\
\hline 11 to 15 years & 0.37 & $0.06-2.64$ & 0.41 & $0.13-1.31$ & 3.16 & $0.79-12.63$ \\
\hline 16 to 20 years & $0.13^{*}$ & $0.02-0.65$ & 0.99 & $0.37-2.64$ & 0.51 & $0.08-3.13$ \\
\hline $21+$ years & 2.95* & $1.09-8.00$ & 1.33 & $0.66-2.68$ & 0.68 & $0.13-2.68$ \\
\hline \multicolumn{7}{|l|}{ Country of origin: } \\
\hline Perú & 0.49 & $0.13-1.78$ & 1.11 & $0.56-2.16$ & $0.40 *$ & $0.19-0.83$ \\
\hline Argentina & 0.58 & $0.25-1.36$ & 1.60 & $0.84-3.04$ & 0.57 & $0.30-1.17$ \\
\hline Bolivia & 0.85 & $0.24-3.01$ & 0.74 & $0.14-3.76$ & 0.62 & $0.10-3.49$ \\
\hline Ecuador & 1.38 & $0.27-6.95$ & 1.09 & $0.20-5.88$ & 0.52 & $0.15-1.74$ \\
\hline GOF test & - & $>0.05$ & - & $>0.05$ & - & $<0.001$ \\
\hline
\end{tabular}

${ }^{*} p$ value $<0.05 .{ }^{* *} p$ value $<0.001$ 
Testing the Latino paradox in Latin America - B. Cabieses et al

living over 20 years in Chile was associated higher chance of disability $(\mathrm{OR}=2.95$, adjusted Wald test $>0.05)$. Country of origin was important to ACCC (Peruvian OR $=0.40)$. Within the Chilean-born, a range of covariates appeared to be significantly associated with health outcomes (Table 3). A partially confounding effect was found between socioeconomic and material factors, since the magnitude of the association between health status and SES was reduced in the presence of material living conditions, but both remained significantly and independently associated with most health outcomes. Multiplicative interaction terms were also found in these models, and the one between age and sex (female) remained significant in the fully adjusted regression $(\mathrm{OR}=1.45)$. Goodness of fit of the logistic models was poor except for those on migration-related factors.

Table 3. Odds Ratio (OR) of presenting different health outcomes in the Chilean-born, adjusted by different sets of SDH separately (CASEN survey 2006) [Significant trends for categorical variables with $>2$ categories appear in grey shade in the table]

\begin{tabular}{|c|c|c|c|c|c|c|}
\hline & \multicolumn{2}{|c|}{ Any disability } & \multicolumn{2}{|c|}{$\begin{array}{l}\text { Any health problem } \\
\text { or accident }\end{array}$} & \multicolumn{2}{|c|}{$\begin{array}{l}\text { Any chronic condition } \\
\text { or cancer }\end{array}$} \\
\hline & OR & $95 \% \mathrm{Cl}$ & OR & $95 \% \mathrm{Cl}$ & OR & $95 \% \mathrm{Cl}$ \\
\hline \multicolumn{7}{|l|}{ DEMOGRAPHIC SDH } \\
\hline Age & $1.05^{*}$ & $1.04-1.05$ & $1.01 *$ & $1.001-1.01$ & $1.06 *$ & $1.05-1.07$ \\
\hline Sex $($ female $=1)$ & 0.94 & $0.89-1.004$ & $1.67 * *$ & $1.52-1.85$ & $1.89 * *$ & $1.58-2.24$ \\
\hline \multicolumn{7}{|l|}{ Marital status: } \\
\hline Single & 1.00 & - & 1.00 & - & 1.00 & - \\
\hline Married & $0.47 * *$ & $0.44-0.51$ & $1.28 * *$ & $1.15-1.43$ & $1.36^{*}$ & $1.11-1.66$ \\
\hline Divorced & $0.69 * *$ & $0.61-0.78$ & $1.31 * *$ & $1.12-1.53$ & $1.51 *$ & $1.15-2.00$ \\
\hline Widow & $0.62 * *$ & $0.56-0.69$ & $1.40 * *$ & $1.06-1.85$ & 1.30 & 0.90-1.87 \\
\hline Ethnicity: any & 0.71 & $0.35-1.44$ & 1.68 & $0.99-2.85$ & $4.76 * *$ & 1.74-13.03 \\
\hline Zone (Rural = 1) & 0.99 & $0.94-1.05$ & $0.74 * *$ & $0.65-0.83$ & 0.89 & 0.73-1.08 \\
\hline \multicolumn{7}{|l|}{ Area: } \\
\hline Northern & 1.00 & - & 1.00 & - & 1.00 & - \\
\hline Central & 1.00 & $0.90-1.12$ & 0.97 & $0.84-1.11$ & 1.25 & $0.96-1.62$ \\
\hline Southern & 1.02 & $0.91-1.14$ & 1.17 & $0.01-1.35$ & $1.43^{*}$ & $1.09-1.88$ \\
\hline GOF test ${ }^{\alpha \beta}$ & - & $<0.001$ & - & $<0.001$ & - & $>0.05$ \\
\hline \multicolumn{7}{|c|}{$\begin{array}{l}\text { SES (adjusted by age, sex and urban/rural) } \\
\text { Educational level: }\end{array}$} \\
\hline No education & $3.70 * *$ & 3.16-4.32 & 1.21 & $0.86-1.69$ & 0.84 & $0.51-1.36$ \\
\hline Primary School & $2.50 * *$ & 2.17-2.88 & 1.08 & $0.92-1.28$ & 1.24 & $0.95-1.63$ \\
\hline High Schoolb & $1.52 * *$ & 1.31-1.75 & 1.03 & $0.89-1.19$ & 1.01 & $0.79-1.30$ \\
\hline Technical level & $1.24 * *$ & $1.05-1.47$ & 1.09 & $0.94-1.27$ & 1.02 & $0.80-1.36$ \\
\hline University level & 1.00 & - & 1.00 & - & 1.00 & - \\
\hline Household income per capita (USD) & $0.68 * *$ & $0.7-0.93$ & 1.07 & $0.89-1.28$ & 0.80 & $0.45-1.98$ \\
\hline Current worker & $0.38 * *$ & $0.27-0.53$ & $0.31 * *$ & $0.26-0.38$ & $0.52 * *$ & $0.38-0.72$ \\
\hline GOF test ${ }^{\alpha \beta \Psi}$ & - & $<0.001$ & - & $<0.001$ & - & $<0.001$ \\
\hline \multicolumn{7}{|c|}{$\begin{array}{l}\text { MATERIAL HOUSEHOLD CONDITIONS (adjusted by age, sex and urban/rural) } \\
\text { Quality Household: }\end{array}$} \\
\hline Acceptable & 1.00 & - & 1.00 & - & 1.00 & - \\
\hline Sub-standard & $1.26^{*}$ & 1.18-1.34 & 1.04 & $0.95-1.15$ & 0.93 & $0.80-1.08$ \\
\hline Unfit & $1.90 *$ & $1.54-2.35$ & 1.20 & $0.83-1.71$ & 0.76 & $0.37-1.57$ \\
\hline Sanitary Index (adequate = 1) & 1.04 & $0.98-1.10$ & $1.14^{*}$ & $1.01-1.30$ & $1.23^{*}$ & $1.01-1.50$ \\
\hline Overcrowding & $0.69 * *$ & $0.64-0.74$ & 0.93 & $0.83-1.04$ & 1.05 & $0.88-1.25$ \\
\hline $\mathrm{HAl}$ & $0.96 *$ & $0.94-0.98$ & $1.02 *$ & $1.007-1.03$ & $1.03^{*}$ & 1.007-1.05 \\
\hline GOF test ${ }^{\alpha \beta}$ & - & $<0.001$ & - & $<0.001$ & - & $<0.001$ \\
\hline
\end{tabular}

${ }^{*} p$ value $<0.05 .{ }^{* *} p$ value $<0.001$. 
Table 4. Odds Ratio (OR) of presenting different health problems if being an international immigrant in Chile, models progressively adjusted by different sets of covariates, CASEN survey 2006

\begin{tabular}{|c|c|c|c|c|}
\hline Health problems & $\begin{array}{c}\text { MODEL } 1 \\
\text { Crude OR of being } \\
\text { immigrant } \\
(95 \% \mathrm{Cl})\end{array}$ & $\begin{array}{c}\text { MODEL } 2 \\
\text { Adjusted OR by } \\
\text { demographics } \\
(95 \% \mathrm{Cl})\end{array}$ & $\begin{array}{c}\text { MODEL } 3 \\
\text { Adjusted OR by } \\
\text { demographics + } \\
\text { SES } \\
(95 \% \mathrm{Cl})\end{array}$ & $\begin{array}{c}\text { MODEL } 4 \\
\text { Adjusted OR by } \\
\text { demographics + } \\
\text { SES + material } \\
(95 \% \mathrm{CI})\end{array}$ \\
\hline Any disability & $\begin{array}{c}0.49 * \\
(0.34-0.70)\end{array}$ & $\begin{array}{c}0.50 * \\
(0.34-0.73)\end{array}$ & $\begin{array}{c}0.67 \\
(0.29-1.54)\end{array}$ & $\begin{array}{c}0.70 \\
(0.30-1.60)\end{array}$ \\
\hline $\begin{array}{l}\text { Any health problem } \\
\text { or accident }\end{array}$ & $\begin{array}{c}0.64^{*} \\
(0.50-0.81)\end{array}$ & $\begin{array}{c}0.63^{*} \\
(0.49-0.80)\end{array}$ & $\begin{array}{c}0.76 \\
(0.52-1.21)\end{array}$ & $\begin{array}{c}0.72 \\
(0.49-1.08)\end{array}$ \\
\hline $\begin{array}{l}\text { Any chronic condition } \\
\text { or cancer }\end{array}$ & $\begin{array}{c}0.65^{*} \\
(0.44-0.95)\end{array}$ & $\begin{array}{c}0.67^{*} \\
(0.42-0.96)\end{array}$ & $\begin{array}{c}0.67 \\
(0.29-1.54)\end{array}$ & $\begin{array}{c}0.70 \\
(0.39-1.60)\end{array}$ \\
\hline
\end{tabular}

${ }^{*} p<0.001$ of being an international immigrant in Chile, weighted logistic regression models.

\section{Testing the Latino paradox}

Analysis was conducted considering the total population sampled in the dataset (Table 4). For every outcome, being a Latino immigrant was a protective factor, after adjusting by demographic covariates. However, the relationship was no longer significant after adjusting for SES. Material living standards did not significantly modify the direction or magnitude of the association between SES and health. Even though the pattern of lower risk among immigrants remains consistent in this analysis, it was no longer statistically significant after adjustment for SES. Hence, the Latino paradox was not observed when comparing Latino immigrants to the Chilean-born population.

\section{Discussion}

\section{Main findings}

A "healthy migrant effect" was found and maintained after controlling for various demographic variables. However, the relative importance of SES for the health of Latino immigrants was statistically substantial. Immigrants in poorer SES showed no difference with the health of the Chilean-population (Table 4). This pattern was observed for the three health problems included in this study.

\section{What is already known and what does this study} add to current knowledge?

Previous analyses of the Latino paradox have demonstrated a healthy migrant effect among
Latino migrants to US and Europe and this may indicate that Latin American culture is more supportive to health than the culture in these host countries ${ }^{11,48}$. However this finding of superior health among predominantly Latin American immigrants to Chile may instead emphasise the significance of health selection effects to understanding healthy migrant effects.

This study supports previous qualitative research in Chile, suggesting that some immigrants live in poor SES and might have urgent health needs ${ }^{25,26,49}$. The healthy migrant effect also disappeared among immigrants living for $>20$ years in Chile. This has been observed in the international literature in the past. In what Rumbaut ${ }^{50}$ called the "paradox of assimilation," the length of time that an immigrant spent in a foreign country has been correlated with an increased risk of a wide range of health problems ${ }^{9}$. In some cases, the rates of poor health may overtake the local rates and immigrants become a severely sick population ${ }^{51}$.

\section{Strengths and limitations}

Four main methodological criticisms regarding the healthy migrant effect and Latino paradox have been discussed in the past: (i) Most studies use the host population as the comparison group and its adequacy as a good counterfactual is debatable, (ii) Most studies examine the health of migrants after they moved to the host country rather than prior to migrating; (iii) Most research relies on self-reported health; (iv) A risk of salmon bias has been described, that is immigrants with poor health return to their countries of origin to be 
treated or to die, which might mislead towards an underestimation of their true ill-health in the foreign country ${ }^{8,18}$.

In this study, the most salient contribution to current knowledge is on the first point. This study considered the Chilean-born as the best counterfactual or comparison groups to Latino migrants and the Latino paradox was not found. The other three points remain as significant constraints of this study and could be tackled in future research, even though self-reported health has proven to be a good predictor of mortality ${ }^{52}$.

\section{Implications and future research}

Chile has become a pole of attraction to Latin American immigrants. There are significant social and health differences ${ }^{53-56}$ and these factors are likely to affect the health of immigrants on arrival and over time. To protect the living conditions and health of immigrants, health policies that ensure equity, culturally appropriate health promotion, and routine assessment are required ${ }^{57}$.

Future research should explore how findings from this study are related to other factors (e.g. stigma, legal status, healthcare provision, etc.). Also, the living conditions and health of immigrants in Chile needs to be analysed prospectively. The Latino paradox could be tested in other countries in order to contribute to the debate on the importance of the adequate counterfactual of migrant populations worldwide.

Acknowledgements: The authors thank (i) The Ministry of Planning, in Chile, for providing us with the CASEN dataset and the guidance received from the field experts; (ii) All the participants from the CASEN 2006 survey who completed this survey; (iii) Professor Mary Maynard, Head of Department of Social Policy and Social Work at the University of York for her valuable feedback; and (iv) Anonymous researchers at different international conferences where preliminary results of this study were presented.

\section{References}

1. Davies M, Adshead F. Closing the gap in a generation: health equity through action on the social determinants of health. An international conference based on the work of the Commission on Social Determinants of
Health, 6-7 november 2008, London. Glob Health Promot 2009; Suppl 1: 7-8; 95-6; 108-9.

2. Davies AA, Mosca D, Frattini C. Migration and health service delivery. World Hosp Health Serv 2010; 46 (3): 5-7.

3. Carballo M, Divino JJ, Zeric D. Migration and health in the European Union. Tropical Medicine \& International Health 1998; 3 (12): 936-44.

4. Feliciano C. Educational selectivity in US immigration: How do immigrants compare to those left behind? Demography 2005; 42 (1): 131-52.

5. Razum O, Zeeb H, Rohrmann S. The 'healthy migrant effect'-not merely a fallacy of inaccurate denominator figures. Int J Epidemiol 2000; 29 (1): 191-2.

6. Fennelly $\mathrm{K}$. The healthy migrant effect. Healthy generations 2007; 5 (3): 1-4.

7. Lu Y. Test of the 'healthy migrant hypothesis': a longitudinal analysis of health selectivity of internal migration in Indonesia. Soc Sci Med 2008; 67 (8): 1331-9.

8. Rubalcava LN, Teruel GM, Thomas D, Goldman N. The healthy migrant effect: New findings from the Mexican family life survey. Am J Public Health 2008 jan; 98 (1): 78-84.

9. Singh GK, Siahpush M. All-cause and cause-specific mortality of immigrants and native born in the United States. Am J Public Health 2001; 91 (3): 392-9.

10. Muennig P, Fahs M. Health status and hospital utilization of recent immigrants to New York City. Prev Med 2002; 35: 225-31.

11. Horton SB, Barker JC. A Latino Oral Health Paradox? Using Ethnography to Specify the Bio-Cultural Factors Behind Epidemiological Models. NAPA Bull 2010 nov 24; 34 (1): 68-83.

12. Cagney KA, Browning CR, Wallace DM. The Latino paradox in neighborhood context: the case of asthma and other respiratory conditions. Am J Public Health 2007; 97 (5): 919-25.

13. Fuentes-Afflick E, Hessol NA, Pérez-Stable EJ. Testing the epidemiologic paradox of low birth weight in Latinos. Arch Pediat Adol Med 1999; 153 (2): 147-53.

14. Abraido-Lanza AF, Dohrenwend BP, Ng-Mak DS, Turner JB. The Latino mortality paradox: a test of the "salmon bias" and healthy migrant hypotheses. Am J Public Health 1999; 89 (10): 1543-8.

15. Franzini L, Ribble JC, Keddie AM. Understanding the Hispanic paradox. Ethnic Dis. [Research Support, NonU.S. Gov't Review]. Autumn 2001; 11 (3): 496-518.

16. Santelli JS, Abraido-Lanza AF, Melnikas AJ. Migration, Acculturation, and Sexual and Reproductive Health of Latino Adolescents. J Adolescent Health 2009; 44 (1): 3-4. 
17. Wolff H, Epiney M, Lourenco AP, Costanza MC, Delieutraz-Marchand J, Andreoli N, et al. Undocumented migrants lack access to pregnancy care and prevention. BMC Public Health 2008 mar 19; 8.

18. Palloni A, Arias E. Paradox lost: Explaining the Hispanic adult mortality advantage. Demography 2004; 41 (3): 385-415.

19. Jolivet A, Cadot E, Florence S, Lesieur S, Lebas J, Chauvin P. Migrant health in French Guiana: Are undocumented immigrants more vulnerable? BMC Public Health 2012; $12(53)$.

20. International Organization for Migration. World Migration report 2011. Communicating effectively about migration World Migration report [serial on the Internet]. 2011; 1: Available from: http://publications.iom. int/bookstore/free/WMR2011_English.pdf.

21. Departamento de Extranjería y Migración. Desarrollo del fenómeno de las migraciones en Chile. Evolución de la gestión gubernamental desde 1990. Ministerio del Interior; 2007 [cited 2008 november]; Available from: http://www.extranjeria.gov.cl/filesapp/migraciones.pdf

22. International Monetary Fund. Report for selected countries and continents: Latin America and the Caribbean GDP ppp in 2010: IMF; 2011 [cited 2011 january]; Available from: http://www.imf.org/external/pubs/ft/ weo/2010/02/weodata/index.aspx

23. Infante A, De la Matta I, López-Acuña D. Reforma de los sistemas de salud en América Latina y el Caribe: Situación y tendencias. Rev Panam Salud Pública 2000; 8: $13-20$

24. Arteaga O, Thollaug S, Nogueira AC, Darras C. [Information for health equity in Chile]. Rev Panam Salud Pública 2002; 11 (5-6): 374-85.

25. Amador M. Forced migration to emerging countries. Case: Chilean society receiving afro-colombian asylum seekers. The 2010 Conference, British Society for Population Studies [serial on the Internet]. 2010: Available from: http://www2.lse.ac.uk/socialPolicy/BSPS/ annualConference/2010/2010 Exeter.aspx-generatedsubheading1.

26. Núnez-Carrasco L, editor. Living on the margins: illness and health care among Peruvian migrants in Chile. First ed. Leisden: Leiden University Medical Centre; 2008.

27. Chilean Ministry of Planning. CASEN 2006. Encuesta de caracterización socioeconómica nacional. Documento metodológico. Santiago: Mideplan; 2006 [cited 2009 october]; Available from: http://www.MIDEPLAN.cl/ casen/index.html

28. Instituto Nacional de Estadistica de Chile. Chile: Proyecciones y Estimaciones de Población. Total País 19502050. Santiago: INE; 2008. Available from: http://www. ine.es/inebase/.

29. Cabieses B, Zitko P, Pinedo R, Espinoza M, Albor C. [How has social status been measured in health research? A review of the international literature]. Rev Panam Salud Pública 2011; 29 (6): 457-68.

30. Filmer D, Pritchett LH. Estimating wealth effects without expenditure data-Or tears: An application to educational enrollments in states of India. Demography 2001 feb; 38 (1): 115-32.

31. Kaiser HF. Coefficient Alpha for a Principal Component and the Kaiser-Guttman Rule. Psychol Rep 1991; 68 (3): 855-8.

32. Townsend P. Deprivation. J Soc Policy 1987; 16: 125-46.

33. Takane Y, Bozdogan H. Akaike Information Criterion (Aic) - Introduction. Psychometrika 1987; 52 (3): 315-.

34. Bozdogan H. Model Selection and Akaike Information Criterion (Aic) - the General-Theory and Its Analytical Extensions. Psychometrika 1987; 52 (3): 345-70.

35. Archer KJ, Lemeshow S. Goodness-of-fit test for a logistic regression model fitted using survey sample data. Stata J 2006; 6 (1): 97-105.

36. Rothman KJ GS, editor. Modern epidemiology. Second ed: Lippincott Williams \& Wilkins, Philadelphia; 1998.

37. Greenwood MJ. Human migration: theory, models, and empirical studies. J Reg Sci 1985; 25 (4): 521-44.

38. Kline DS. Push and pull factors in international nurse migration. J Nurs Scholarsh [Review] 2003; 35 (2): 10711.

39. Recio DM. Editorial: The push and pull in migration. ANPHI Pap 1973; 8 (3): 2.

40. Liang Z, Chunyu MD, Zhuang G, Ye W. Cumulative causation, market transition, and emigration from China. Ajs. [Research Support, N.I.H., Extramural Research Support, Non-U.S. Gov't Research Support, U.S. Gov't, Non-P.H.S.] 2008; 114 (3): 706-37.

41. Massey DS. Social structure, household strategies, and the cumulative causation of migration. Popul Index 1990 Spring; 56 (1): 3-26.

42. Fussell E, Massey DS. The limits to cumulative causation: international migration from Mexican urban areas. Demography. [Research Support, Non-U.S. Gov't Research Support, U.S. Gov't, P.H.S.]. 2004 feb; 41 (1): 151-71.

43. Pickles A. Commentary: Trajectories, selection and cumulative causation. International Journal of Epidemiology [Comment] 2007; 36 (3): 548-9.

44. Pyle J. Globalization, transnational migration, and gendered care work: introduction. Globalizations 2006; 3 (3): 283-95.

45. Radcliffe SA. Culture and development in a globalising world : geographies, actors, and paradigms. Milton Park, Abingdon, Oxon; New York: Routledge; 2006. 
Testing the Latino paradox in Latin America - B. Cabieses et al

46. Macpherson DW, Gushulak BD, Macdonald L. Health and foreign policy: influences of migration and population mobility. Bull World Health Organ 2007; 85 (3): 200-6.

47. Yu HJ, Cumberland WG. Logistic regression models for analysis of multistage survey data. American Statistical Association-1996 Proceedings of the Section on Survey Research Methods, Vols I and Ii. 1996: 468-73.

48. Abraido-Lanza AF, White K, Armbrister AN, Link BG. Health status, activity limitations, and disability in work and housework among Latinos and non-Latinos with arthritis: An analysis of national data. Arthrit RheumArthr 2006; 55 (3): 442-50.

49. International Organization for Migration, Ministerio de Salud de Chile. Migración y salud en Chile. Estudio de Salud Global en poblacion immigrante en Chile. 2008: Available from: http://www.oimconosur.org/notas/buscador.php?nota=716.

50. Rumbaut RG. Introduction: Immigration and incorporation. Sociol Perspect. 1997; 40 (3): 333-8.

51. Fernando Riosmena JAD. A Tale of Three Paradoxes: The Weak Socioeconomic Gradients in Health Among Hispanic Immigrants and Their Relation to the Hispa- nic Health Paradox and Negative Acculturation Aging, Health, and Longevity in the Mexican-Origin Population 2012; 2: 95-111.

52. Galobardes B, Lynch J, Smith GD. Measuring socioeconomic position in health research. Br Med Bull 2007; 81-82: 21-37.

53. Arteaga O, Astorga I, Pinto AM. [Inequalities in public health care provision in Chile]. Cad Saude Publica 2002; 18 (4): 1053-66.

54. Chilean Ministry of Health. Impacto del piloto AUGE en la atencion de salud. Biblioteca del Congreso Nacional de Chile. 2004: Available from: http://www.bcn.cl/ carpeta_temas/temas_portada.2005-10-27.7644862447/ documentos_pdf.2005-10-28.2320771530

55. Vega J, Bedregal P, Jadue L, Delgado I. [Gender inequity in the access to health care in Chile]. Rev Med Chile 2003; 131 (6): 669-78.

56. Mardones F. [Inequality of health care for the elderly in Chile]. Rev Med Chile 2004; 132 (7): 865-72.

57. Lassetter JH, Callister LC. The Impact of Migration on the Health of Voluntary Migrants in Western Societies A Review of the Literature. J Transcult Nurs 2009; 20 (1): 93-104. 